\title{
La géographie est-elle une science? Introduction aux problèmes de codification dans le traitement automatique de l'information géographique
}

\section{Marc Boyé}

Volume 14, numéro 32, 1970

URI : https://id.erudit.org/iderudit/020907ar

DOI : https://doi.org/10.7202/020907ar

Aller au sommaire du numéro

Éditeur(s)

Département de géographie de l'Université Laval

ISSN

0007-9766 (imprimé)

1708-8968 (numérique)

Découvrir la revue

Citer cet article

Boyé, M. (1970). La géographie est-elle une science? Introduction aux problèmes de codification dans le traitement automatique de l'information géographique. Cahiers de géographie du Québec, 14(32), 157-169. https://doi.org/10.7202/020907ar
Résumé de l'article

Le présent article est à la fois une introduction épistémologique à la géographie et une approche sémantique du langage, fort complexe, qu'emploient les géographes. Son but est d'inviter à la réflexion sur les problèmes que pose la codification de l'information géographique pour un traitement automatique.

Comme toutes les disciplines chargées de gérer et de présenter un « corps de savoir ", la géographie est aujourd'hui confrontée à l'accumulation accélérée de la masse documentaire qu'elle utilise. Les éléments qui composent son corps de savoir viennent pour la plupart d'autres sciences et d'autres disciplines, d'un degré de complexité moindre, qui lui fournissent des informations concernant la Terre et les Hommes. Le rôle du géographe est de synthétiser ces apports en vue de rendre compte de la répartition des faits physiques ou humains considérés à la surface du globe et d'en produire une expression cartographique ; son point de vue est celui d'un généraliste. Toutefois, la géographie ne s'intéresse pas aux faits sur le seul plan statistique ; elle considère encore leurs rapports et leur genèse, voire leur devenir en ce qu'ils sont, eux aussi, susceptibles de représentation cartographique. Il n'y a donc pas, à proprement parler, d'information géographique, mais une manière géographique de dresser la synthèse d'informations de provenances diverses. Or, chaque source d'information a son langage propre, de sorte que le langage géographique procède pour une grande part d'emprunts et ne dispose d'un langage spécifique qu'à partir d'un certain degré de synthèse.

Il en résulte que, n'étant ni une science ni une discipline scientifique, même lorsqu'elle s'équipe de méthodes pour " percevoir et pénétrer » du nouveau, la géographie ne peut pas se plier à une codification unique, sous la forme d'un thésaurus de mots-clés. Il lui faut au moins trois ordres de codes aptes à un jeu de combinaisons :

1 - Un code géographique, pour la localisation à la surface du globe ; un système de coordonnées par exemple ;

2 - Un code taxonomique, c'est-à-dire un vocabulaire des « maîtres-mots » qui portent l'esprit même de la préoccupation géographique et constituent la classification typologique propre à la discipline :

3 - Un code syntaxique, où notamment les emprunts faits à d'autres langages seraient à faire jouer comme des données grammaticales.

Par la logique même de l'argumentation, des notions comme science, discipline, connaissance, corps de savoir ont trouvé l'occasion d'être précisées.
Ce document est protégé par la loi sur le droit d'auteur. L'utilisation des services d'Érudit (y compris la reproduction) est assujettie à sa politique d'utilisation que vous pouvez consulter en ligne.

https://apropos.erudit.org/fr/usagers/politique-dutilisation/ 


\title{
LA GÉOGRAPHIE EST-ELLE UNE SCIENCE ?
}

\author{
Introduction aux problèmes de codification dans le traitement \\ automatique de l'information géographique *
}

par

\section{Marc BOYÉ}

Centre d'études de géographie tropicale, 33-Talence, France

II y a déjà une vingtaine d'années, considérant l'essor scientifique prodigieux et accéléré - A. CAILLEUX $(1951, b)$ proposait une classification des sciences, différente de celle d'Auguste COMTE (1830) en ce qu'elle introduit les idées nouvelles «... relatives à l'univers, à l'espace et au temps ". Il distingue deux grands groupes:

les sciences sans localisation (mathématique, physique, chimie, etc.) qui font abstraction des lieux et des époques, étudient des propriétés et les fonctions et traitent surtout des grandeurs;

- les sciences à localisation qui étudient des faits et des comportements, donc s'intéressent volontiers aux objets (êtres et choses).

Ces dernières peuvent être soit à définition temporelle (histoire, archéologie, etc.), soit à définition à la fois temporelle et spatiale (astronomie, géologie, etc.), soit à définition spatiale. La géographie, avec ses divers champs d'étude, est classée dans ce dernier sous-groupe (voir notamment le tableau 2, p. 8, op. cit.).

Méditant successivement sur la pensée et le mot dans l'acte de recherche, sur l'évolution quantitative du langage, sur le rôle des articles (défini, indéfini) dans l'expression des dénombrements entiers, fondement de la systématique, A. CAILLEUX $(1951 \mathrm{a}, 1953,1954)$ en est venu à se préoccuper du problème de l'organisation des publications scientifiques. II l'a fait dans

*De nombreux collègues et collaborateurs ont bien voulu lire et commenter le présent article ; qu'ils en soient cordialement remerciés. Mais j'ai une dette particulière à l'égard de MM. A. CAILLEUX, P. GOUROU, et J.P. SIMÉON. Le premier, actuellement professeur à l'université Laval de Québec, n'a cessé depuis 22 ans de me faire part de ses propres réflexions sur le sujet et $\mathrm{m}$ 'a toujours ouvert libéralement sa bibliothèque. Le deuxième, professeur au Collège de France, par ses aimables remarques, m'a confirmé dans ce que je propose. Quant au troisième, professeur de philosophie au Lycée mixte d'État de Talence, il a eu la patience de relever mes insuffisances en philosophie. Qu'ils veuillent trouver ici l'expression de ma respectueuse gratitude. Je tiens aussi à remercier $M$. $L$. PAPY, doyen de la Faculté des Lettres et Sciences humaines de Bordeaux, qui m'a vigoureusement encouragé à publier cet essai. 
le cadre de I'Union géographique internationale, au titre de président de la Commission de morphologie périglaciaire (A. CAILLEUX, 1956). II y livre ainsi sa préoccupation: "Dans l'océan débordant de nos publications, il nous faut des ilôts, des bouées, des radeaux de sauvetage. Sinon, le document risque de submerger le documenté ».

La nécessité de nouveaux langages en découle inévitablement car il s'agit de perfectionner et d'adapter les moyens d'expression et de mémorisation à la vitesse d'évolution des techniques et à l'accumulation des connaissances qu'elles apportent. Sur cette voie, les géologues pétroliers sont peut-être les plus en avance ; bien que très spécialisé, un ouvrage récent, comme celui de BYRAMJEE, COMBAZ et sept autres co-auteurs (1969) sur les méthodes modernes de traitement de l'information géologique sur ordinateurs, montre l'esprit dans lequel il faut oeuvrer.

Les géographes de l'université Laval à Québec ( $H$. DORION, 1964 et R. LEBLOND, 1969) ont inauguré de très intéressantes expériences documentaires à l'aide du système MIRACODE (Microfilm Information Retrieval Access Code) mis au point par la Société Recordak. Ils travaillent actuellement à indexer et à microfilmer ce qu'ils nomment "les cartes non autonomes", c'est-à-dire les cartes thématiques logées dans les livres, revues et thèses d'intérêt géographique. Ils ont déjà proposé un premier essai de mode de codification où ils ont choisi, en fonction de l'appareil qu'il utilise, une classification hiérarchique (du général au particulier) complétée par un thésaurus des mots-clés, c'est-à-dire un lexique descriptif codé. Ils font très justement remarquer que le développement d'un mode de codification est un exercice sémantique.

J'ai été amené, à mon tour, à méditer sur ce problème par les difficultés d'une gestion moderne de la connaissance géographique par les méthodes de l'information. Notamment, l'établissement d'un thésaurus des mots-clés nécessaires à la géographie, même réduite par convention au monde tropical chaud et humide ${ }^{1}$, doit tenir compte - et en même temps rendre compte d'un degré élevé de complexité.

Qu'est-ce donc que la géographie?

J'observe d'abord que l'école géographique française, issue, par le biais de I'histoire 2, du monde mental façonné par la Faculté des Lettres, aurait tendance à ranger la géographie parmi les sciences. Pourquoi?

1 Comme c'est l'objectif de la division de la documentation au Centre d'études de géographie tropicale (C.N.R.S.) à Bordeaux.

2 Dans le Dictionnaire des sciences, lettres et arts de M. N. BOUILLET (1884), on trouve à l'article "science " une classification en cinq groupes: sciences philosophiques, sciences historiques, sciences mathématiques, sciences physiques et naturelles, sciences occultes (sic). La géographie est rangée dans le second, aux côtés de l'histoire, de la chronologie, de l'ethnographie, de la biographie et même du blason! 
Le caractère original de la géographie française, ou plutôt l'accent qu'elle met dans son oeuvre, est de vouloir présenter de la face de la terre une description synthétique où concourent les aspects physiques et les activités humaines, notamment celles qui relèvent du moteur économique. Elle ne limite pas son projet à une sorte de descriptive des faits qui ne serait au total qu'un art. Elle veut aussi participer à leur explication en considérant leur évolution afin de rendre compte de l'état présent qui en serait le résultat. Elle envisage donc le côté génétique de ce qu'elle étudie. C'est là que le géographe se sent et se dit servir une science puisque, en effet, il coordonne un ensemble de notions relatives à un objet déterminé. Son oeuvre de coordination est parfaitement illustrée par le style même des grandes thèses de géographie, le plus souvent consacrées à l'étude globale d'une région. On peut même dire que la géographie régionale est une conception spécifiquement française. Peu d'autres cultures en effet conçoivent la géographie sous un angle aussi généraliste.

Dans son introduction à un manuel récent, G. VIERS (1967) rappelle que la géographie est «... essentiellement la science de l'organisation de l'espace ॥. II fait remarquer que l'organisation de l'espace est un concept plus global que celui d'aménagement de l'espace, puisque tous les éléments naturels ou non y sont évoqués. En fait, la géographie est appelée à opérer la synthèse de données fournies par d'autres sciences: les sciences naturelles pour une bonne part, les sciences exactes parfois, les sciences expérimentales plus fréquemment, surtout de nos jours; toutefois ce sont les sciences dites humaines qu'il manipule le plus couramment car elles sont, mieux que les autres, adaptées à sa structure mentale et littéraire. À ce point de la réflexion il vaut de relire le jugement que portait A. de LAPPARENT (1896) dans la préface de ses Leçons de géographie physique. Estimant la géologie susceptible d'apporter aux études de géographie la rigueur d'analyse convenable, à condition de la dépouiller de " . . . son aridité traditionnelle », il constatait en même temps que "... les traditions de l'enseignement littéraire n'ont préparé ni les maîtres ni les disciplines ... . à recevoir la somme et la nature des connaissances scientifiques exigées des géographes de qualité. Pour désigner ces connaissances, le géographe emploie spontanément des vocables tels que sciences annexes ou bien sciences auxiliaires qui marquent clairement le complexe de supériorité que son effort de synthèse nourrit en lui ; ceci étant à titre phénoménologique et sans aucun jugement de valeur ! II est évident que les tenants de ces sciences ne peuvent pas l'entendre de même façon. Les plus spécialisés d'entre eux saisissent mal la prétention scientifique de la géographie. D'aucuns y voient même une sorte de poétique, au sens grec du mot, c'est-à-dire une construction idéale où les faits de répartition dans la nature (formes de reliefs, paysages ...) et l'écologie humaine sont en effet présentés, parfois, comme obéissant à des règles, à la manière dont la versification obéit à celles de la métrique. À cet égard les querelles entre géographes déterministes et ceux qui tiennent à d'autres dialectiques donnent à penser. On constate en tout cas que les théories antithétiques tiennent une bonne place dans les discussions entre géographes.

Il est toutefois des sciences, ou plutôt des lignes de pensée scientifique dont les démarches sont plus proches - et leurs servants plus compréhensifs - des démarches de la géographie ; spécialement l'histoire et la géologie, 
qui est aussi une histoire. Cela vient de ce qu'elles sont également tenues à une conception généraliste. Dans une adresse présidentielle à l'Assemblée générale de l'Association des géologues du Bassin de Paris, P. RAT (1969), cherchant à définir l'esprit et les démarches de la paléogéographie, s'en est référé à la géographie actuelle en la caractérisant ainsi : "On peut dire qu'il n'y a pas de faits géographiques, mais une manière géographique de considérer chaque ensemble de faits $"$.

Une seconde remarque me vient ici : il me semble que l'on commet ordinairement sur le mot science un contre-sens. On lui fait volontiers signifier connaissance. Par exemple, on dit un homme de science pour caractériser celui qui emploie les méthodes scientifiques à la fois dans le raisonnement, l'expérimentation et aussi dans le mode d'expression des conclusions ou des résultats acquis. À cet égard certains géographes sont des scientifiques; je pense aux géographes économistes ou bien aux géomorphologues. Or ils le sont dans la mesure même où ils se spécialisent en se coulant dans le moule mental de telle ou telle science. Reconnaissons que c'est gênant, à la fois pour les classer puisqu'ils sont en même temps spécialistes et généralistes, mais aussi pour eux-mêmes car ils doivent mener deux opérations: I'une d'ordre analytique, l'autre d'ordre synthétique. Comparons avec l'expression puits de science. Elle désigne quelqu'un dont la somme de connaissances est à la fois considérable dans son étendue et précise dans sa gestion. À ce titre, tout géographe digne de ce nom se doit d'être un puits de science. En France, il est formé à cela ; il suffit de considérer, pour s'en convaincre, un programme d'agrégation. On dit encore - et l'on s'en émerveille - les progrès de la science. L'ambiguité est ici éclatante. Certains y voient le fruit du labeur des scientifiques et l'honneur ainsi rendu à l'esprit humain. D'autres spéculent sur les conséquences économiques et sociales d'un tel progrès pour l'utilisation qu'on peut en faire, y compris la commercialisation des résultats. Mais le plus grand nombre considère surtout dans le progrès l'accumulation des connaissances et le cheminement progressif vers la vérité, c'est-à-dire cet état idéal de la connaissance où s'éclaireraient enfin les rapports entre les produits de la raison et les intuitions où se mêlent les données sensorielles et les réactions affectives.

Qu'est-ce alors que la science? II convient de s'en référer à l'étymologie. Science vient du latin scire: savoir. Là est l'origine du contre-sens. Nous faisons du verbe savoir un substantif: le savoir ! II s'agit donc très manifestement de lui faire signifier moins l'acquisition que l'acquis de la connaisance. Nous le traitons plus comme un verbe d'état que comme un verbe d'action. Tous les dictionnaires, qui utilisent le langage de la philosophie positive, sont très explicites à cet égard. Or le scire latin, comme le ci ou le $k i$ sanscrit, proviennent d'un vieux fond indo-européen, certains pensent même pré-indo-européen, où la racine $k i$ porte un sens très dynamique: à la fois percevoir et pénétrer. Est donc véritablement une science ce qui, par les processus intellectuels mis en jeu, par les procédés expérimentaux employés, permet d'accéder à la connaissance, de la perfec- 
tionner, de la confirmer, ce que A. CAILLEUX (1963) appelle «la prise d'information efficace ", voire de la constituer par l'invention. Mais ce n'est pas la connaissance elle-même !

Dès lors qu'ayant acquis la connaissance, il s'agit de gérer le corps de savoir ${ }^{3}$ ainsi rassemblé, il ne devrait plus être question de science mais de discipline. L'ordonnancement même de la connaissance, que nous appelons classification est certes de présentation analytique mais il répond en fait à une préoccupation d'ordre synthétique. Il s'agit en effet non seulement de présenter les résultats obtenus dans un ordre logique satisfaisant pour l'esprit (surtout s'il est cartésien), mais encore de proposer l'ensemble du corps de savoir, ainsi établi, dans son aspect global, cohérent et en même temps susceptible de prolongement. A. CAILLEUX ${ }^{4}$ suggère que "sciences et disciplines vivent comme des plantes ". Elles s'accroissent, se diversifient en se ramifiant. Elles sont en effet douées d'un dynamisme propre orienté vers la recherche du renouveau et au bout de chaque rameau d'une classification, toute possibilité de bourgeonnement reste ouverte. Nous ne nous en rendons pas forcément compte et si chacune des sciences particulières poursuit son effort jusque là c'est qu'elle éprouve le besoin légitime de vérifier sa méthode, nécessairement analytique puisque sa vocation est de discerner, quitte d'ailleurs à se transformer en discipline puisque, au moins un bilan des connaissances acquises est indispensable au progrès de la recherche. Au-delà, lorsqu'il faut confronter les résultats des diverses sciences, à fortiori combiner les apports des disciplines correspondantes, ou simplement les comparer, alors un modèle synthétique devient nécessaire. L'expérience prouve que c'est souvent un corps de doctrine qui en tient lieu, avec les risques à consentir, notamment ceux de la dogmatique. C'est pourquoi il convient de réserver le terme de discipline à ce stade de gestion de la connaissance.

Il en découle que la géographie n'est pas une science; elle est une discipline, c'est-à-dire une forme d'étude qui exige une systématique. Des auteurs anciens en ont eu l'intuition; par exemple F. S. BEUDANT (1833). Dans l'introduction générale à son traité de physique, le minéralogiste qu'il était à éprouvé le besoin de fournir lui aussi une classification des sciences qui l'intéressaient. Deux groupes sont proposés : les sciences mathématiques et les sciences physiques. II convient de posséder les premières avant d'aborder les secondes en raison, dit cet auteur, de la "fécondité ordinaire " du calcul qui peut conduire à "... une multitude de conséquences ». La géographie se trouve classée dans le second groupe, mais elle est donnée comme divisée en plusieurs parties :

1. La géographie théorique ou mathématique : c'est celle qui considère la terre comme un corps géométrique, doué de mouvements et à repérer par rapport aux astres afin de "... fixer exactement .. . la position des lieux", pour en marquer la situation relative sur des plans et des cartes. Dès ici la préoccupation cartographique ressort clairement.

3 L'expression nous est proposée par notre collègue J. P. SIMÉON, professeur de philosophie au Lycée Victor Louis de Talence, qui a bien voulu lire et critiquer le présent travail.

4 In litteris. 
2. La géographie physique est présentée comme subdivisée en deux branches :

- L'une ne reçoit pas de nom mais elle considère : la configuration du terrain, la position des îles et celle des montagnes, la direction des vallées, la forme des plaines, etc. II s'agit bien de l'étude du relief; ce n'est pas encore ce que nous nommerions aujourd'hui géomorphologie ; c'est déjà une bonne partie de ce que les auteurs anglo-saxons désignent par physiographie.

- L'autre branche étudie les rapports entre ces faits et la constitution du terrain, la nature et la position des couches minérales. BEUDANT indique un nom curieux : la géognosie et précise aussitôt, qu'ainsi considérée, cette partie de la géographie rentre dans le domaine de l'histoire naturelle avec la géologie, les climats, la distribution des animaux et des végétaux. C'est pourquoi, plus loin dans sa classification, il distingue successivement une géographie botanique et une géographie zoologique; ce que nous traduirons volontiers par biogéographie.

Il est évident que BEUDANT avait le sens de la variété des domaines envisagés par la géographie et de leurs relations; ce qui importe c'est que, pour lui, la géographie est essentiellement appliquée aux faits de distribution et à leur localisation sur le globe terrestre.

Telle est, en effet, la systématique propre à la géographie : rendre compte de la répartition et représenter cartographiquement les faits humains, aussi bien que physiques, que l'on peut enregistrer à la surface du globe ${ }^{5}$. Singulière ambition! II faut bien voir, qu'étendant son regard à la fois sur l'ensemble de la planète matérielle et sur l'écoumène, le géographe embrasse, forcément, tous les corps de savoir. Non pas que la géographie commande les sciences qui les ont produits, mais parce que toute science contribue à la constituer, en ce sens que la réflexion et l'expérimentation scientifiques, en étendant le champ du connu concernant la terre et les hommes, étendent du même coup le domaine de la géographie. De sorte qu'il en est un peu du géographe comme du médecin généraliste, selon que me le suggère $P$. GOUROU (in litteris), sa culture doit être vaste et son attention constamment en alerte, mais il doit aussi savoir consulter les spécialistes, sans se départir de l'esprit critique nécessaire à la synthèse, qui est son objectif.

II me paraît en résulter une conséquence ; c'est ma troisième remarque : de même que certaines sciences sont, par leur mouvement propre, amenées à se constituer en disciplines - ainsi de la géologie par exemple - de même la géographie est conduite à s'équiper scientifiquement. II lui arrive en effet de se fournir de concepts originaux ; par exemple celui de croissance urbaine qui est l'aspect dynamique de la géographie des villes que l'on peut aussi bien saisir dans son historique que dans sa phénoménologie actuelle

5 Voir P. RAT, 1969, op. cit., page 7. 
et dont l'intérêt prospectif est évident. II lui arrive de se munir de méthodes appropriées : par exemple tout ce que les géographes ont rassemblé, voire inventé, pour une étude quantitative sur l'évolution des versants. II reste que ces démarches de caractère scientifique sont faites en vue d'établir des classifications qui soient propres à la géographie. Dans le cas de la géologie comme dans celui de la géographie, la confusion des genres prête à réflexion. Prenons le cas, partiel donc plus limité, de la géomorphologie. Son but est de mieux décrire et classer telle forme du terrain en exposant sa raison d'être et son devenir. La part faite dans une telle étude aux processus en cause identifie, de ce point de vue, la géomorphologie à ce que les géologues appellent la géologie dynamique. Cependant la spécificité géographique d'une étude des formes du relief comporte que soit précisé l'environnement biogéographique, ou seulement climatologique, non seulement à titre de cadre mais encore en raison de la convergence géographique des divers facteurs entrant en ligne de compte. C'est en ce sens qu'il existe une géomorphologie climatique, elle-même incluse dans une géographie zonale ou azonale selon l'expression lancée par Emm. de MARTONNE (1946). De cette conception on trouvera une illustration dans le plan de recherches proposé par O. RIBEIRO (1954) pour l'étude des paysages à inselberge. Sept grandes rubriques sont envisagées : définition, nature des roches, la forme, les dépôts associés, les relations avec le réseau hydrographique, celles avec le climat (et les paléoclimats), enfin les processus. On voit par là combien est déjà complexe le champ d'étude de la géomorphologie. J. TRICART (1965) l'a d'ailleurs fort bien montré dans son "schéma des mécanismes de causalité en géomorphologie " où est représenté le jeu des interactions du point de vue dynamique, compte tenu de la notion d'évolution, c'est-à-dire du caractère irréversible du temps. J'ai moi-même eu l'occasion d'aborder à plusieurs reprises la question de l'héritage en géomorphologie, jusqu'à proposer la notion d'adaptation généalogique de formes de surface à des topographies souterraines ayant affecté des terrains anciens (M. BOYÉ, M. MOULINE et autres, 1968).

Ces perspectives invitent au raisonnement à plusieurs dimensions comme I'a si souvent rappelé dans son oeuvre P. TEILHARD DE CHARDIN ${ }^{6}$.

De ce fait il apparaît nécessaire que le géomorphologue soit un homme de science, mais la géomorphologie demeure une discipline en ce qu'elle réunit les apports de plusieurs sciences. Prenons un exemple : soit à étudier le modelé des reliefs latéritiques cuirassés en pays de savanes.

Le cadre géographique apparaît clairement dans la condition limitative exprimée par pays de savanes. Or savane est un concept biogéographique. Certes un tel terme implique la dimension zonale et impose la zonalité au titre de l'ordre supérieur dans une classification. II n'en existe pas moins des savanes azonales que l'on traite soit comme des exceptions à la règle - la savanisation anthropique par exemple - soit comme des nuances à apporter et donc relevant d'un ordre de classification subordonné. L'esprit géographique d'une telle classification est donc respecté et l'on admet que le concept de savane soit annexé au vocabulaire géographique au titre d'un type de paysage qui se situe sous certaines latitudes et non

6 Par exemple: La vision du passé, 1957 ; l'univers à trois infinis, page 317. 
sous d'autres. Il y a là un cas d'emprunt démonstratif! C'est un emprunt non à une science mais à une discipline de type écologique ; la biogéographie elle-même fait appel à diverses sciences: climatologie, pédologie, biologie ...., sans compter tout ce qu'il y a à considérer des actions de I'homme, ne serait-ce que le facteur pyrogène qu'il représente, comme le savent bien tous les tropicalistes (voir par exemple Y. MONNIER, 1968).

Examinons les mots reliefs et modelé. Ils appartiennent en propre au vocabulaire géomorphologique, surtout lorsqu'ils sont accolés. Ils ne sont cependant pas du même ordre et impliquent une subordination d'échelle du second au premier. Relief, en français classique, désigne un reste, un résidu. En géomorphologie le mot devrait signifier strictement: " ce que l'érosion n'a pas enlevé ॥. C'est d'ailleurs le sens qu'il a gardé en anglais ( $A$. CAILLEUX, in litteris). L'expression relief résiduel est donc une tautologie. Pratiquement dans la littérature géographique française usuelle, le mot introduit une notion de topographie et invite à une descriptive de caractère géométrique. C'est un concept statique d'un certain ensemble où les données structurales exercent souvent une influence prépondérante. Modelé, se réfère aux détails de façonnement expressifs d'une histoire de la forme, telle qu'on peut - ou croit pouvoir - la déduire de l'observation de traits de détail. C'est donc un concept à la fois plus ordonné à la plastique des formes et plus évolutionniste. L'analyse à ce niveau exige un comportement scientifique, depuis la manière de relever tel trait jusqu'au traitement statistique des données recueillies, car ces traits constituent des sous-ensembles à examiner. D'autre part ils comportent des aspects dynamiques, soit de l'ordre de l'évidence à consigner, soit de l'ordre d'hypothèses à vérifier par la confrontation des faits avec le raisonnement. De fait l'étude d'un modelé exige en priorité la considération des processus impliqués. Or il s'agit presque toujours d'une combinaison de processus et l'étude de chacun d'eux relève d'une science déterminée : mécanique des matériaux et des fluides, chimie, biologie, thermodynamique ... pour ne citer que les majeures. Si bien qu'au stade de la présentation des résultats le modelé est déjà, à son ordre, une synthèse.

Si l'on considère maintenant, dans l'exemple proposé, les ordres inférieurs de la définition catégorielle: latéritiques cuirassés, on remarque d'abord qu'on emploie pour eux des adjectifs et non plus des substantifs ${ }^{7}$. Cette indication grammaticale prouve qu'il peut entrer dans une classification non seulement un vocabulaire mais aussi une syntaxe. Par ailleurs ces deux termes font immédiatement apercevoir toute la gamme des vocabulaires spécialisés à emprunter à d'autres disciplines, notamment à la pédologie. Laissons de côté la notion de latérite qui recouvre tellement de domaines scientifiques différents que la discussion mériterait à elle seule un traité ${ }^{8}$. Prenons seulement la notion de cuirasse. En tant qu'image d'un certain type de surface topographique elle pourrait être directement annexée au vocabulaire géomorphologique sur les types de reliefs latéritiques. C'est d'ailleurs ce que font les géographes tropicaux lorsqu'ils généralisent le mot guinéen

7 A. CAILLEUX (in litteris) me fait observer, au passage, que la langue française exprime ici, grammaticalement, une nuance de pensée, mieux que la langue anglaise, prise dans le carcan de ses substantifs en apposition.

8 Voir par exemple R. MAIGNIEN (1966). 
bowal ${ }^{9}$. Mais lorsque l'on se souvient que l'effort des géologues minéralogistes et des pédologues nous apprend que certaines cuirasses dites "d'accumulation relative" des sesquioxydes (il vaudrait mieux dire : concentration) se constituent à une certaine profondeur dans un profil d'altération, alors que d'autres (cuirasses de nappe ou de versant) résultent d'apports latéraux de fer, on réalise comment de sérieuses erreurs d'interprétation ont pu être commises et combien, par conséquent, le géomorphologue est dépendant, pour sa synthèse, d'une foule de données scientifiques (c.f. : G. ROUGERIE, 1959).

S'il n'avait qu'à rassembler et coordonner ces données, comme le maçon qui n'a pas à se préoccuper de la manière dont les briques qu'il emploie ont été cuites, il pourrait se contenter d'être un compilateur intelligent et un statisticien un peu exercé. Mais dès lors que les données de la chimie, de la physique, de la biologie (microbiologie entre autres) qu'il utilise sont discutables ou incertaines, il est contraint à un travail critique. C'est en cela qu'il se doit d'être lui-même un scientifique et qu'il le devient quand, par défaut de l'une des sciences concernées, les circonstances le conduisent à imaginer, tester et mettre en oeuvre une méthode nouvelle, ne serait-ce qu'une technique de diagnose. Je peux le dire d'expérience, car 25 années de recherches géomorphologiques me l'ont constamment démontré. C'est au moment où il en arrive à déposer ses conclusions que le géomorphologue doit faire oeuvre de géographe ; il lui faut alors :

1. harmoniser les diverses terminologies utilisées, sans les trahir ${ }^{10}$, afin de présenter les résultats de manière cohérente par rapport aux sciences dont ils relèvent; c'est l'aspect syntaxique de son effort ;

2. insérer la logique de son raisonnement dans le cadre spatiotemporel qui définit l'environnement actuel — ou passé — des formes qu'il a étudiées, afin de dégager des types et une classification selon un vocabulaire propre :

3. enfin situer dans le monde, ou la partie du monde considérée, ce qu'il vient de définir, afin d'en produire, éventuellement, une expression cartographique.

Le cas du géomorphologue n'est pas unique parmi les géographes. Quelle que soit leur orientation préférentielle (géographie physique, humaine ou économique) ceux-ci rencontrent tous les mêmes problèmes et doivent

9 Bowal désigne, en Guinée, des affleurements de cuirasse dénudée, souvent en buttes résiduelles à sommet plat et corniches abruptes de latérite.

10 C'est pourquoi, en géographie moins qu'ailleurs, une équipe de recherche ne doit pas tant réunir divers spécialistes au titre d'une fédération de compétences, mais bien plutôt combiner au creuset de la recherche elle-même, à la fois les méthodes et les formes de pensée, de manière à obtenir plus vite des résultats plus complets et à s'assurer, lors de la formulation des conclusions, qu'il ne se glisse pas des erreurs de jugement dues à des ignorances d'ordre sémantique. 
adopter la même démarche. La conclusion est claire: LA GÉOGRAPHIE N'EST PAS UNE SCIENCE; même lorsqu'elle incorpore de la connaissance scientifique. ELLE EST UNE DISCIPLINE ! Elle n'est même pas une discipline scientifique puisque son objet dépasse la seule connaissance des liens entre nécessités naturelles. Elle fait appel non seulement à des sciences diverses mais aussi à diverses disciplines d'un degré de complexité moindre que la sienne. On pourrait, à la limite, dire qu'elle intègre, dans la sphère géographique qui lui est propre, non seulement de la connaissance scientifique, mais encore de la connaissance philosophique (N. BERDAIEFF, 1936) dès lors qu'elle veut, à juste titre, considérer l'homme, ses problèmes écologiques, son devenir économique et social, voire ses aspirations spirituelles en ce que l'activité qu'il déploie donne lieu à des phénomènes de répartition et marque, par des faits, l'emprise de l'espèce humaine sur le globe. La géographie est oecuménique au sens étymologique premier (cf. MAX SORRE, 1943, T. I.).

Tout récemment, P. CLAVAL (1970) s'est intéressé aux influences de la géographie physique et des sciences naturelles qu'elle utilise, sur les concepts et les méthodes de la géographie humaine. II fait très justement remarquer que ce sont deux disciplines traditionnelles associées mais, quant au fond, il les estime autonomes. II montre en effet que la géographie humaine a subi, de ce point de vue, une influence non négligeable mais qui a été "... indirecte, inconsciente presque ». Autrement dit à l'intérieur même de la géographie il y aurait diversité de disciplines.

II en résulte qu'un thésaurus de géographie ne peut pas se restreindre à un vocabulaire de mots-clés. II lui faut, forcément, le jeu de trois ordres de codes, pour que l'expression codée soit convenablement synthétique et commode à utiliser, même dans le cas où l'on choisirait de restreindre l'emploi à la seule gestion automatique d'une documentation bibliographique.

1. Un code géographique, composé de signaux de situation qui traduiraient par leur agencement même dans la mémoire de la machine, la position géographique. En y réfléchissant bien, on est amené à concevoir un système de coordonnées qui, par un curieux retour de l'esprit humain sur lui-même, ramène au système des coordonnées géographiques en latitudes et longitudes. C'est ici aux mathématiciens programmateurs de métier de répondre à la demande.

2. Un code taxonomique, c'est-à-dire un vocabulaire, non pas de mots-clés, mais des maîtres-mots de la géographie. Mot-clé ne convient pas ici car, dans le langage de l'informatique, il désigne à la fois l'idée qu'il porte, exprimée de manière conventionnellement concise, et l'artifice par lequel il se situe comme descripteur ou sous-descripteur dans une hiérarchisation, à base décimale par exemple. La clé en effet est ordonnée à la serrure qu'elle permet d'ouvrir. Théoriquement réalisable en géographie, un tel vocabulaire nécessiterait un recensement exhaustif des mots-clés. II serait alors énorme, trop complexe et donc déraisonnable. I faut donc se restreindre aux maîtres-mots. Par maître-mots il faut entendre les mots ou les locutions qui portent l'esprit même de la préoccupation géographique. Il s'agit à l'évidence des termes de la classification typologique recherchée, dont la caractéristique doit être de pouvoir donner lieu à la cartographie. 
Même restreint de la sorte, le vocabulaire des géographes est en partie fait d'emprunts par voie fréquente d'annexion. Encore faut-il s'assurer que lors de l'annexion le sens du mot emprunté n'a pas dévié, n'a pas dérivé. S'il a dérivé, alors l'emploi d'un adjectif ou d'un complément de nom approprié devrait assez aisément préciser l'intention purement géographique. Ainsi du mot faille qui, chez les mineurs et chez les géologues désigne une discontinuité, par rupture et déplacement, dans la stratigraphie ou les structures magmatiques, alors que pour les géographes il évoque plutôt l'influence de ce type d'accident tectonique sur le modelé, à la fois quant à la localisation et au style cassé de la forme de terrain étudiée. Par exemple on dira, selon le besoin : "escarpement de faille ", "relief de ligne de faille ", "faille exhumée par l'érosion ". De la sorte pourrait se constituer la gamme souhaitable de sous-descripteurs. C'est ici aux géographes à prononcer leurs options.

3. Enfin un code syntaxique ; il pourrait être représenté par les vocabulaires directement empruntés (en tout ou partie) aux sciences concernées. Les mots de ces vocabulaires seraient à faire manipuler par la machine comme autant de données grammaticales que de besoin. En première approximation, il semble possible d'envisager des combinaisons à la manière dont procèdent les langues agglutinantes. C'est ici aux informaticiens de montrer les ressources de leur art, à condition que les géographes leur expriment clairement leurs besoins et que soient consultés, voire adjoints aux équipes de travail, les divers spécialistes intéressés. J'irai jusqu'à penser que le concours de logiciens et de linguistes s'impose.

BERDAIEFF, N. (1936), Cinq méditations sur l'existence. Paris, Aubier-Montaigne, édit., Coll. Philosophie de l'esprit. 208 pages.

BEUDANT, F. S. (1833), Traité élémentaire de physique. Paris, chez Verdière, 5 e édition, 700 pages.

BOUILLET, M. N. (1884), Dictionnaire universel des sciences, lettres et arts. Paris, Hachette, 14e édition. 1817 pages.

BOYÉ, M. ; MOULINE, M. ; PRATVIEL, L. ; VIGUIER, C. ; (1968), "Relations entre la forme des cours inférieurs de la Garonne et de la Dordogne et les topographies souterraines des terrains tertiaires ". Revue de Géomorphologie Dynamique, $\mathrm{n}^{\circ} 2$, pages 83-91.

BYRAMJEE, M. : COMBAZ, M. : DUPUY, M.: ÉTIENNE, M. FONCK, M.: GONNON, M. : JEKHOWSKY, M. de; LEROY, M. ; SOURISSE, M.; (1969), Méthodes modernes de traitement de l'information géologique sur ordinateur. Paris, Éditions Technip, 144 pages.

CAILLEUX, A. (1951,a), "La pensée et le mot dans l'acte de recherche. "Revue générale des sciences, t. $58, n^{\circ}$ 5-6.

CAILLEUX, A. $(1951, b)$, "Lieux, époques, espace, temps et classification des sciences ". Revue générale des sciences, t. 58, $\mathrm{n}^{\circ}$ 9-10, p. 397-306, Paris.

CAILLEUX, A. (1953), "Évolution quantitative du langage. " Bulletin S.P.F., t. 50, $\mathrm{n}^{\circ}$ 9-10, pages 505-514

CAILLEUX, A. (1954), "Articles, dénombrements entiers et systématique. " Revue des questions scientifiques, $5 \mathrm{e}$ série, t. 15 , pages $404-418$.

CALLEUX, A. (1956), Introduction sur l'organisation des publications scientifiques. Rapport de la Commission de morphologie périglaciaire de I'U.G.I.; Congrès international, Rio de Janeiro 1956. Lódż, Biuletyn Peryglacjalny, $n^{\circ} 4$, pages 5.7

CAILLEUX, A. (1963), "Expérience, intention et information efficace. " Revue de synthèse, T. 83, Sections généralités, 3e série, $n^{\circ} 29$.

CLAVAL, P. (1970), "L'influence de la géographie physique et de la géographie matérielle sur les concepts et les méthodes de la géographie humaine. " Annales de Géographie, T. 41, fasc. 2, pages 113-122.

DORION, H. (1964), L'utilisation du système MIRACODE dans le traitement de l'information géographique. Québec, Centre de documentation, université Laval. Document ronéotypé

LAPPARENT, A. de (1898), Leçons de géographie physique. Paris, Masson et Cie, 2 e édition, 718 pages.

MAIGNIEN, R. (1966), Compte-rendu de recherches sur les latérites. Paris, Publication UNESCO, Série recherches sur les ressources naturelles, IV. 157 pages.

MARTONNE, Emm. de (1946), "Géographie zonale, la zone tropicale. " Annales de géographie, $\mathrm{n}^{\circ} 1$. pages 1-18. 
MONNIER, Y. (1968), Les effets des feux de brousse sur une savane préforestière de Cóte d'lvoire. Études éburnéennes, IX, Direction de la recherche scientifique, Ministère de I'Éducation nationale de la République de Côte d'Ivoire, Abidjan. 260 pages.

RAT, P. (1969), "Esprit et démarches de la paléogéographie. Exemples dans le Bassin parisien. " Bulletin de la société géologique de France, (7), XI, pages 5-12.

RIBEIRO, O. (1954), Paysages à inselberge. Perth, Proceedings, Pan Indian Scientific Congress, Section F, pages 29-30.

ROUGERIE, G. (1959), "Latéritisation et pédogénèse intertropicales. 》 Information géographique, 23e année, $n^{\circ} 5$, pages 199-205.

SORRE, Max (1947), Les fondements de la géographie humaine, Tome l, les fondements biologiques; essai d'une écologie de l'homme. Paris, Armand Colin, 2 e édition, 447 pages.

TEILHARD DE CHARDIN, P. (1957), La vision du passé. Paris, Éditions du Seuil, 391 pages.

TRICART, J. (1965), "Schéma des mécanismes de causalité en géomorphologie." Annales de géographie, $74 \mathrm{e}$ année, pages $322-326$.

VIERS, G. (1967), Éléments de géomorphologie. Paris, Fernand Nathan, Collection FAC, 5.20 pages.

\section{RÉSUMÉ}

Le présent article est à la fois une introduction épistémologique à la géographie et une approche sémantique du langage, fort complexe, qu'emploient les géographes. Son but est d'inviter à la réflexion sur les problèmes que pose la codification de l'information géographique pour un traitement automatique.

Comme toutes les disciplines chargées de gérer et de présenter un "corps de savoir », la géographie est aujourd'hui confrontée à l'accumulation accélérée de la masse documentaire qu'elle utilise. Les éléments qui composent son corps de savoir viennent pour la plupart d'autres sciences et d'autres disciplines, d'un degré de complexité moindre, qui lui fournissent des informations concernant la Terre et les Hommes. Le rôle du géographe est de synthétiser ces apports en vue de rendre compte de la répartition des faits physiques ou humains considérés à la surface du globe et d'en produire une expression cartographique; son point de vue est celui d'un généraliste. Toutefois, la géographie ne s'intéresse pas aux faits sur le seul plan statistique; elle considère encore leurs rapports et leur genèse, voire leur devenir en ce qu'ils sont, eux aussi, susceptibles de représentation cartographique. II n'y a donc pas, à proprement parler, d'information géographique, mais une manière géographique de dresser la synthèse d'informations de provenances diverses. Or, chaque source d'information a son langage propre, de sorte que le langage géographique procède pour une grande part d'emprunts et ne dispose d'un langage spécifique qu'à partir d'un certain degré de synthèse.

II en résulte que, n'étant ni une science ni une discipline scientifique, même lorsqu'elle s'équipe de méthodes pour "percevoir et pénétrer » du nouveau, la géographie ne peut pas se plier à une codification unique, sous la forme d'un thésaurus de mots-clés. II lui faut au moins trois ordres de codes aptes à un jeu de combinaisons:

1 - Un code géographique, pour la localisation à la surface du globe ; un système de coordonnées par exemple ;

2 - Un code taxonomique, c'est-à-dire un vocabulaire des "maîtres-mots " qui portent l'esprit même de la préoccupation géographique et constituent la classification typologique propre à la discipline ;

3 - Un code syntaxique, où notamment les emprunts faits à d'autres langages seraient à faire jouer comme des données grammaticales.

Par la logique même de l'argumentation, des notions comme science, discipline, connaissance, corps de savoir ont trouvé l'occasion d'être précisées.

\section{ABSTRACT}

The present article is both an epistomologic introduction to geography and a semantic approach to the language, often extremely complex, that geographers employ. Its purpose is to invite reflection upon the problems posed by the codification of geographic information for automatic handling. 
As is the case with all scientific disciplines responsible for assembling and presenting a "body of learning ", geography today is confronted with the task of digesting the pyramiding mountains of documentary material which it must use. The elements which form its body of learning come for the most part from other sciences and other spheres of study, of a lesser degree of complexity, which furnish it with information concerning Man and the Earth. The geographer's role is to synthesize these contributions so as to account for the distribution of physical or human factors spread out over the surface of the globe and with them produce a cartographic picture; his approach is that of an analyst. Still, geography does not concern itself with facts solely in a statistical sense; it also considers their relationships and their origins and, what's more, the potential of these too in turn to be transformed into cartographic representations. Thus, strictly speaking, there is no geographic information as such but rather a geographic manner to develop a synthesis of the information obtained from diverse sources.

Now, the problem is precisely here ; each information source has its own language so that geographic language is for most part formed of borrowed terms and does not possess its own terminology except from a certain advanced point of synthesis.

The result is that, being neither a science nor a scientific discipline even when it is equipped with tools for "grasping and penetrating " the new, geography cannot rely upon a unique codification method such as, for example, a thesaurus of key terms. It requires at least three types of codes suited for a set of different combinations :

1 - A geographic code, for the localization of points on the surface of the globe ; a coordinates system, for example ;

2 - A taxonomic code, that is to say, a vocabulary of "key terms " imbued with the very spirit of geography and which constitutes the typological classification belonging to the discipline itself;

3 - A syntax code, where the terms borrowed from other languages will be employed as grammatical data.

For the principle of discussion, ideas connoted by such words as science, discipline, knowledge, body of learning have been clarified. 